\title{
A SMART AND DISTRIBUTED MEASUREMENT SYSTEM TO ACQUIRE AND ANALYZE MECHANICAL MOTION PARAMETERS
}

\author{
J.M. Dias Pereira ${ }^{1,2)}$, Vítor Viegas ${ }^{1,2)}$, Octavian Postolache ${ }^{3,4)}$, Pedro Silva Girão ${ }^{2)}$ \\ 1) Instituto Politécnico de Setúbal, ESTSetúbal/LabIM, , 2910-761 Setúbal, Portugal, (هdias.pereira@estsetubal.ips.pt, +351 265 790000) \\ 2) Instituto de Telecomunicações DEEC/IST/UTL, Av. Rovisco Pais, 1, 1049-001 Lisbon, Portugal \\ 3) Instituto de Telecomunicações/IT-IUL, Av. das Forças Armadas, 1649-026 Lisbon, Portugal \\ 4) Instituto Universitário de Lisboa ( ISCTE-IUL), Av. das Forças Armadas, 1649-026 Lisbon, Portugal
}

\begin{abstract}
This paper presents a low-cost and smart measurement system to acquire and analyze mechanical motion parameters. The measurement system integrates several measuring nodes that include one or more triaxial accelerometers, a temperature sensor, a data acquisition unit and a wireless communication unit. Particular attention was dedicated to measurement system accuracy and compensation of measurement errors caused by power supply voltage variations, by temperature variations and by accelerometers' misalignments. Mathematical relationships for error compensation were derived and software routines for measurement system configuration, data acquisition, data processing, and self-testing purposes were developed. The paper includes several simulation and experimental results obtained from an assembled prototype based on a crank-piston mechanism.
\end{abstract}

Keywords: measurement system, kinematic variables measurements, accelerometers, error compensation, accuracy.

(C) 2013 Polish Academy of Sciences. All rights reserved

\section{Introduction}

Amplitude, phase and frequency measurements of kinematic variables, namely position, velocity and acceleration, are essential to a huge number of applications that include condition monitoring of machinery devices. This paper presents a low-cost measurement system to acquire and analyze mechanical motion parameters. The proposed measurement system includes several measuring nodes (MNs) with sensing, data conditioning, analog to digital conversion and data transmission capabilities. It is important to underline that wireless communication capability of the $\mathrm{MN}$ is essential in this type of application since data acquisition from moving devices does not allow usually the use of wired connections. Moreover, it is important to refer that the distributed topology of the measurement system allows simultaneous measurements of kinematic variables, namely position, velocity and acceleration, among others, at different measurement points, being possible to establish crosscorrelations between measurement data acquired in different MNs [1-4]. Measurement system fault detection routines can be easily implemented by using the measurement data of each $\mathrm{MN}$, considering, for example, the expected measuring range of each variable or the crosscorrelation that is expected between different measuring quantities [5-7]. Obviously, this advantage can only be achieved if the theoretical model of the system is known or if the mechanical device under test had been previously characterized. Concerning monitoring purposes, it must be underlined that abnormal variation of kinematic parameters indicates potential failures of the mechanical devices under analysis [8-10]. Thus, monitoring of mechanical devices, particularly the ones that are submitted to hard working conditions, is 
essential to schedule predictive maintenance [11] and then to improve mechanical systems' reliability.

In order to improve measurement accuracy, several error compensation techniques are presented [12-14]. In this context, steady state measurements of the mechanical device, namely tilt measurements of mechanical elements, can be used to compensate misalignment measurement errors since it is particularly important to extract from the accelerometer signal the component that is associated with gravity acceleration [15]. Otherwise, the acceleration component that is associated with the mechanical motion will be mixed with tilt components that result from the relative positioning of the mechanical elements associated with each MN. Other errors, like the ones caused by temperature variations that can substantially affect measurement accuracy, are also compensated; otherwise, it will be impossible to distinguish mechanical motion parameter variations from the ones that are caused by temperature variations.

Regarding applications of the proposed measurement system, it can be said that it can be used in a large number of industries that employ power transmission units and components, like linear actuators, clutches and automotive timing chain drive systems used to drive camshafts in auto engines [16]. Other typical examples that can illustrate systems' applications in industrial environments occur in the synchronization that must exist between different running shafts of a computer numerical control (CNC) machinery [4], [17] or in the coordination of robots that exhibit the capability to execute movements of multiple axes [18-19]. Others types of applications are related with vibration measurements that are very important to evaluate the performance of industrial plants, namely the measurement of piping structures, motors and control valve vibrations, among others, which are essential to schedule corrective and predictive maintenance tasks based on regular or continuous monitoring results [20].

It is important to underline that there exist some commercial solutions for identical measurement purposes, however, these solutions are very expensive, proprietary and their flexibility to define new measurement configurations and data processing algorithms, suitable for specific applications, is very low [21].

This paper is organized in five sections. The present one is the introduction, section two describes the measurement system, namely its hardware and software parts, section three is dedicated to measurement error compensation, section four includes simulation and experimental results and the last section, section five, is dedicated to conclusions.

\section{System description}

This section includes the description of the measurement system hardware and software components, underlining their main characteristics.

\subsection{Hardware}

The hardware of the measurement system includes several measuring nodes (MNs) and a coordinator node $(\mathrm{CN})$.

It is important to underline that the number of sensors in each $\mathrm{MN}$ is selected according to users' requirements and mechanical device under test (MDUT) characteristics. Fig. 1 represents a typical $\mathrm{MN}$ block diagram that includes two accelerometer devices and a temperature sensor. Additional sensing elements can be included in each $\mathrm{MN}$ according to the type and the number of quantities that must be measured. Measurement tests were performed with other sensor types, namely, vibration sensors, pressure sensors and encoders. 
used to find the set of piston-crank dimensional parameters (l,r) and the rotation frequency (f) that minimizes the difference between measurement and curve fitting data.

From the experimental data that was obtained it possible to verify that there is a signal-tonoise ratio of the measurement signal almost equal to $19 \mathrm{~dB}$.

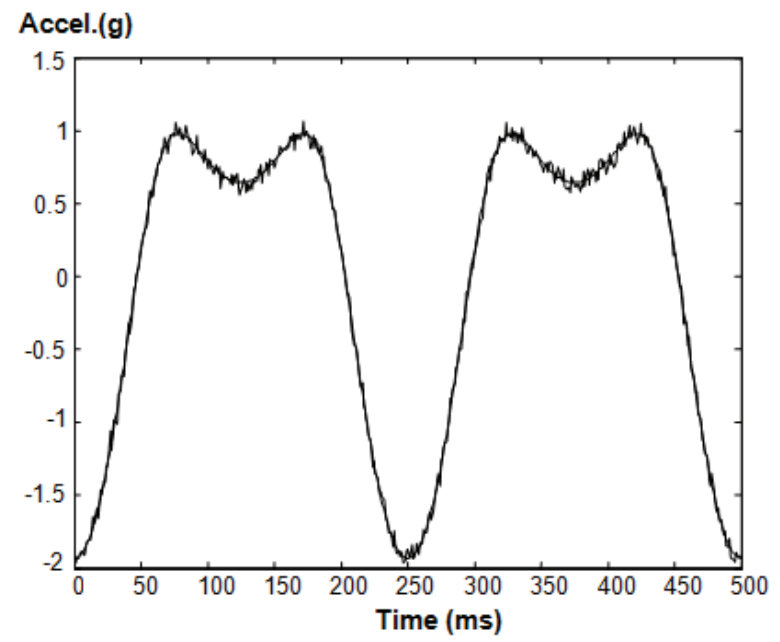

(a)

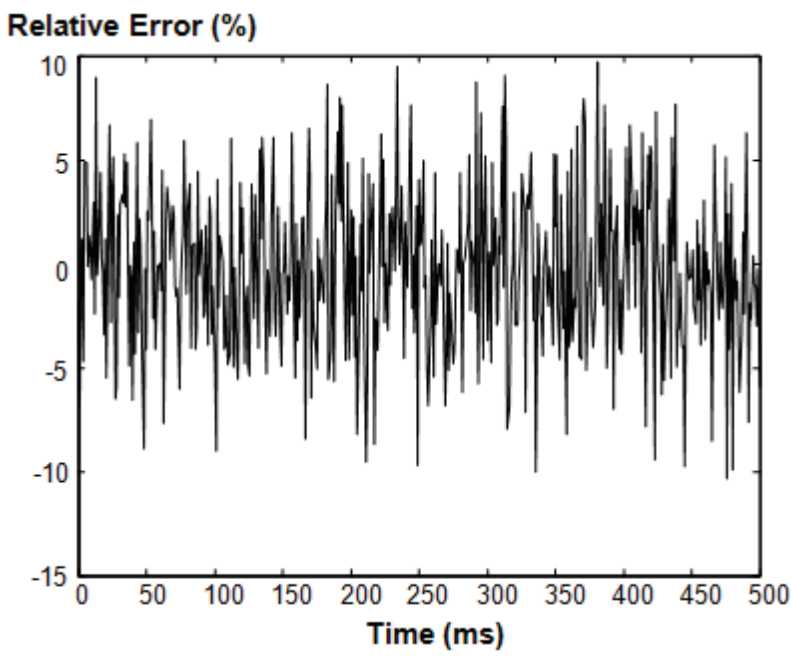

(b)

Fig. 11. (a) Measured and curve fitting data that were obtained for the following set of parameters: $1=4 \mathrm{~cm}$, $\mathrm{r}=2 \mathrm{~cm}$ and freq=4 Hz; (b) Relative error between measurement curve fitting data.

It is important to underline that dithering, noise shaping, digital filtering, and decimation techniques can be used to improve the signal-to-noise ratio and the resolution of the measurement system, obviously, at the expense of input signal bandwidth [43].

\section{Conclusion}

A wireless measurement system based on a set of different measuring nodes that include one or more accelerometers, a temperature sensor, a data acquisition unit and a wireless communication unit was presented. Curve fitting techniques were successfully used to evaluate the deviation between experimental data and the theoretical data derived for the kinematic equations of the MDUT. Particular attention was dedicated to the measurement system accuracy and to the compensation of the measurement errors caused by power supply voltage variations, by temperature variations, and by accelerometers' or mechanical system misalignments. Experimental tests were performed using a crank-piston mechanism and a 
measurement system with three measuring nodes. The experimental results that were obtained confirmed the theoretical expectations based on the kinematic equations of the mechanical device under test. Finally, it is important to underline that the accuracy values that were obtained can be substantially improved, since the low-cost prototype that was used to perform the experimental tests was designed for teaching purposes and not for accurate measurements.

\section{References}

[1] Abuthakeer, S.S., Mohanram, P.V., Mohankumar, G. (2011). The Effect of Spindle Vibration on Surface Roughness of Workspiece in Dry Turning Using ANN. International Lean Thinking, Vol. 2, Issue 2.

[2] Tobias, S. et al. (2005). Influence of Surface Roughness on Friction During Metal Forming Processes. Journal of Materials Processing Technology. 159, 9-16.

[3] Li, Y., Lin, J., Wang, X., Liao, Y. (2011). Multiple-axis synchronization evaluation for CNC machine tool based on sensor less measurement. In Proceedings of IEEE International Symposium on Assembly and Manufacturing (ISAM), Vol.1, 1-5.

[4] Albarbar, A., Mekid, S., Starr, A. and Pietruszkiewicz, R. (2008). Suitability of MS Accelerometers for Condition Monitoring: An experimental study. Sensors, Issue (8), 784-799.

[5] Liming, Wu, Junxiu, L., Ji, Zhang, Yuling, L. (2009). The Intelligent Reconfigurable Measuring Node. Proceedings of the International Conference on International Conference on Computational Intelligence and Security (CIS '09), Vol. 1, 570-573.

[6] Chao, Fan, Zhang, De-xian, Hong-liang, Fu, Yi-tao, Liang (2010). Design of the Measurement Node of the Grain Quantity Monitoring System Based on the CAN-bus. Proceedings of the International Conference on Challenges in Environmental Science and Computer Engineering (CESCE), Vol. 1, 211214.

[7] Peng, Faya (2005). Design considerations for a distributed test system. In Proceedings of IEEE Autotestcon, 235-239.

[8] Aidemark, J., Folkesson, P., Karlsson, J. (2005). A framework for node-level fault tolerance in distributed real-time systems. In Proceedings of International Conference on Dependable Systems and Networks, 656665.

[9] Ananthanarayanan, S.P., Szymczyk, C., Goldenberg, A.A. (1992). Identification of kinematic parameters of multiple closed chain robotic manipulators working in coordination. In Proceedings of IEEE International Conference on Robotics and Automation, Vol. 1, 358-363.

[10] Zhang, Bin et al. (2011). A Probabilistic Fault Detection Approach: Application to Bearing Fault Detection. IEEE Transactions on Industrial Electronics, Vol. 58, Issue 5, 2011-2018.

[11] Dayly, J.W., Riley, W.F., McConnell (1984). Instrumentation for Engineering Measurements. New York, NY: John Wiley \& Sons.

[12] Wanli, Liu, Xinghu, Q, Yonggang, Y (2007). Self-Calibration and Error Compensation of Flexible Coordinate Measuring Robot. In Proceedings of International Conference on Mechatronics and Automation, 2489-2494.

[13] Kermorgant, O., Folio, D., Chaumette, F. (2010). A new sensor self-calibration framework from velocity measurements. In Proceeding of Conference on Robotics and Automation, 1524 - 1529.

[14] Li, X, et al (2011). An error compensation method for multi-axis machining based on the actual contour measurement. In Proceedings of IEEE International Symposium on IEEE International Symposium on Assembly and Manufacturing, 1-5.

[15] Alloca, J.A., Stuart, A. (1984). Transducers Theory \& Applications. Reston Publishing Company, Inc., Prentice-Hall.

[16] Tsubakimoto Chain CO., Automotive Parts Products (April, 2012). http://tsubakimoto.com.

[17] Mahrenholtz, O., Bontcheva, N., Iankov, R. (2005). Influence of surface roughness on friction during metal forming processes. J. Mater. Process. Technology, No. 159, 9-16.

[18] Quigley, M., Brewer, R., Soundararaj, S.P., Pradeep, V., Quoc Le, Ng, A.Y (2010). Low-cost accelerometers for robotic manipulator perception. In Proceedings of Internation Conference on Intelligent Robots and Systems (IROS), Vol. 1, $6168-6174$.

[19] Renk, E.L., Rizzo, M., Collins, W., Lee, F., Bernstein, D.S.. Calibrating a triaxial accelerometermagnetometer - using robotic actuation for sensor reorientation during data collection. IEEE Control Systems, Vol. 25 , Issue $6,86-95$. 
[20] Dias Pereira, J.M., Postolache, O., César, M.L., Girão, P.S (2012). A Smart Sensing System to Analyze Piping Vibrations in Industrial Installations. In Proceedings of the International Conf. on Sensing Technology (ICST 2012), Vol. 1, 7-14.

[21] Midé Technology. Slam Stick ${ }^{\mathrm{TM}}$ Vibration Recorder $\quad(2012 \quad$ Sept.). http//:www.digikey.com/uk/en/ph/mide/slamstick.html.

[22] Dallas Semiconductor, DS1809 Dallastat ${ }^{\mathrm{TM}}$ - 64-position digital potentiometer (2010 Sept.) http://www.datasheets.maxim-ic.com/en/ds/DS1809.pdf.

[23] National Instruments. An Overview of IEEE 1451.4 Transducer Electronic Data Sheets (2010 Sept.). http://www.standards.ieee.org/develop/regauth/tut/teds.pdf.

[24] National Instruments. Data Acquisition Board- 9215 (2010 Sept.). http://www.sine.ni.com/nips/cds/view/p/lang/en/nid/208793.

[25] Abdelhamid, M.K. (2003). Towards Smarter Measurement Systems. In Proceedings of Conference IMAC-XXI: Conference \& Exposition on Structural Dynamics. https://www.sem.org/Proceedings/ ConfPapersPaperID $=25721$

[26] Yurish, S.Y., Gomes, M.T. (2004). Smart Sensors and MEMS. II Mathematics, Physics and Chemistry, Vol. 181, NATO Sciences Series, Kluwer Academic Publishers.

[27] Analog Devices, iMEMS Accelerometer $\quad$ ADXL330 $\quad(2010 \quad$ Sept.). http://www.datasheetarchive.com/ADXL330KCPZ-datasheet.html.

[28] Smartec, SMT 160-30 Temperature Sensor (2010 Sept.). http://www.smartec-sensors.com.

[29] International Frequency Sensor Association, Universal Frequency-to-Digital Converter (Sept. 2010). http://www.sensorsportal.com.

[30] Microchip Technology Inc., PIC16F87X Microcontrolle (2010 Sept.). http://www.microchip.com.

[31] MDFLY Electronics, Wireless Bluetooth TTL Transceiver Module (Sept. 2010). http://www.mdfly.com

[32] Texas Instruments, UAF42-Universal Active Filter datasheet. http://www.ti.com/lit/ds/symlink/uaf42.pdf.

[33] Pereira, D.P., Girão, P.S., Postolache, O. (2001). Fitting Transducer Characteristics to Measured Data. IEEE Instrumentation \& Measurement Magazine, 4(4), 26-39.

[34] National Instruments, LabVIEW 9.0. http://www.ni.com/labview .

[35] Cherry, Speed \& Proximity Sensors, Geartooth Speed and Direction Sensor, part \# SD100203 (2010 Sept.). http://www.cherrycorp.com.

[36] Analog Devices (2010). Accelerometer Design and Applications. Application Note AN-1057. http://www.analog.com.

[37] Kuorilehto, M., et al. (2007). Ultra-Low Energy Wireless Sensor Networks in Practice: theory, realization and deployment. Finland: John Wiley and Sons, Ltd..

[38] Pereira, J.M., Postolache, O., Girão, P.S. (2007). A Digitally Programmable A/D Converter for Smart Sensors Applications. IEEE Transactions on Instrumentation and Measurement, Vol.56, No.1, 158-163.

[39] IIT Kharagpur (2010), Introduction to Belt Drives, module 13, version 2 ME (2010 Sept.) http://www.nptel.iitm.ac.in/courses.

[40] Taylor, C.F. (1985). The Internal Combustion Engine in Theory and Practice, Vol. 1 \& 2, 2nd Edition. MIT Press.

[41] Danfoss, Variable speed driver, VLT 2800 Series (Sept. 2010). http://www.ledcontrols.co.uk.

[42] Lagarias, J.C., Reeds, J.A., Wright, M.H., Wright, P.E. (1998). Convergence Properties of the Nelder-Mead Simplex Method in Low Dimensions. SIAM Journal of Optimization, Vol. 9, No. 1, 112-147.

[43] Dias Pereira, J.M., Serra, A. Cruz, Girão, P. (2000). Flexible ADC: a Dither and Oversampling Based Solution to Improve the Performance of ADC Systems. In Proceedings of IMEKO World Congress 2000. Wien, Austria, 103-108. 